\title{
Parcourir les histoires de l'art non-linéaires : les études du British Black Art par Sophie Orlando
}

\author{
Ella S. Mills
}

Traducteur : Thomas Golsenne et révisée par Chloé Maillet

\section{OpenEdition}

Journals

Édition électronique

URL : http://journals.openedition.org/imagesrevues/4875

DOI : $10.4000 /$ imagesrevues.4875

ISSN : 1778-3801

Éditeur :

Centre d'Histoire et Théorie des Arts, Groupe d'Anthropologie Historique de l'Occident Médiéval Laboratoire d'Anthropologie Sociale, UMR 8210 Anthropologie et Histoire des Mondes Antiques

Référence électronique

Ella S. Mills, « Parcourir les histoires de l'art non-linéaires : les études du British Black Art par Sophie Orlando », Images Re-vues [En ligne], Hors-série 6 | 2018, mis en ligne le 31 juillet 2018, consulté le 30 janvier 2021. URL : http://journals.openedition.org/imagesrevues/4875 ; DOI : https://doi.org/ 10.4000/imagesrevues.4875

Ce document a été généré automatiquement le 30 janvier 2021.

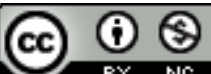

Images Re-vues est mise à disposition selon les termes de la Licence Creative Commons Attribution Pas d'Utilisation Commerciale 4.0 International. 


\title{
Parcourir les histoires de l'art non- linéaires : les études du British Black Art par Sophie Orlando ${ }^{1}$
}

\author{
Ella S. Mills \\ Traduction : Thomas Golsenne et révisée par Chloé Maillet
}

1 J'aurais voulu que le livre de Sophie Orlando, British Black Art. L'histoire de l'art occidental en débat, soit publié une dizaine d'années plus tôt. Je commençai alors mes recherches sur ce qui est désormais connu (au moins dans le domaine de l'art britannique) sous le nom du mouvement des Black Arts des années $1980^{2}$. C'est le mouvement défini une décennie entière de l'histoire de l'art britannique, produisant quelques unes des œuvres d'art visuel les plus puissantes de ces dernières années par des artistes trop nombreux pour être énumérés ici. Dans son livre, Orlando choisit de mettre en valeur des œuvres de Lubaina Himid, Eddie Chambers, Rasheed Araeen, Sonia Boyce et Chila Kumari Burman. Le mouvement Black Art était traversé par une incroyable énergie de passion créatrice, avec des individus, des collectifs et des collaborateurs, dont beaucoup d'entre eux étaient aussi des animateurs, "des individus qui prenaient en charge plusieurs rôles : des artistes qui sont aussi commissaires, qui écrivent, qui défendent, et qui font aussi le travail d'archive $»^{3}$. Moment profondément complexe de l'histoire de l'art, parcouru de débats au long cours et de questions politiques aussi bien " internes » qu'« externes » au mouvement, la pratique des artistes du British Black Art a manqué pendant des années d'une analyse critique substantielle et soutenue et d'un discours bien renseigné qui pouvait inscrire cette pratique dans le paysage plus large de l'histoire de l'art et de la mémoire culturelle britanniques. En termes de documentation académique accessible, il aura fallu attendre, comme le rappelle Orlando, la publication en 2001 de Shades of Black: Assembling Black Art in 1980s Britain pour pouvoir lire une tentative collective spécialisée visant à revisiter les années $1980^{4}$. Le livre était issu d'un colloque de deux jours à Duke University en Caroline du Nord. Orlando remarque que les organisateurs britanniques - le photographe David A. Bailey, l'artiste Sonia Boyce et le professeur de littérature anglaise à Duke University Ian Baucom - signalaient «l'urgence d'interroger désormais les productions de manière critique pour aller au- 
delà de l'analyse sociologique des conditions d'émergence du mouvement en tenant compte de l'effort de documentation entrepris depuis la fin des années 1980 par les acteurs mêmes de ce mouvement $»^{5}$. Malgré la demande formulée par de nombreux participants impliqués dans Shades of Black en 2001 (artistes, curateurs, responsables d'institutions d'art, chercheur·se-s), ce n'est que depuis ces toutes dernières années que l'engagement critique a connu une dynamique et une accélération significatives. Le livre d'Orlando est une réponse à ces appels lancés pendant Shades of Black et participe à ce moment clé de l'activité réflexive et analytique.

2 Un des premiers points à remarquer concernant le livre d'Orlando, qui peut particulièrement intéresser celles et ceux qui ne sont pas familiere's de cette période de l'histoire de l'art britannique, est son titre. Même s'il étudie brièvement des artistes africain'e-s et asiatiques qui vivaient et travaillaient dans les années 1960 et 1970, le travail d'Orlando se concentre principalement sur le mouvement du British Black Art des années 1980 et son activité jusqu'à la fin des années 1990. Bien qu'elle ne soit pas clairement spécifiée dans le titre de son livre, British Black Art. L'histoire de l'art occidental en débat, cette attention portée à deux décennies est justifiée par l'affirmation selon laquelle le «Black Art » est un terme directement associé aux années 1980 (et au-delà ) » Les artistes liés aux diasporas afro-caraibéennes ou asiatiques sont à distinguer du «Black art » ou du British Black art, le mouvement artistique né dans les années 1980 » , « le terme Black proposant une posture politique commune aux migrants et enfants de migrants sur un territoire postcolonial et Européen $»^{6}$.

"Black », en termes artistiques, apparaissait de fait comme une affirmation politique pour les artistes plus jeunes, émergeant dans les années 1980, la première génération des «artistes British Black» née's ou élevée's au Royaume-Uni et qui se sont diplômée's dans des institutions d'art britanniques. Mais pour beaucoup d'artistes africain'e-s ou asiatiques de la génération précédente, née's dans des pays colonisés et qui vinrent au Royaume-Uni en tant qu'étudiante's plus âgée's, il s'agissait d'une appellation troublante qui ne véhiculait pas la même signification.

Il faut ajouter à cette contextualisation, pour les lecteurs-rices novices sur cette période de l'histoire de l'art, que la dynamique de l'activité créatrice des British Black Artists pendant les années 1980 était réputée difficile à définir et à discuter pour les critiques d'art. Le théoricien britannique Stuart Hall décrivit ce moment, dans sa conférence à Shades of Black, de cette manière: "loin d'être un phénomène unifié, cohérent ou organisé, ce "mouvement" (si l'on peut appeler ainsi quelque chose d'aussi diffus) [...] défie les espaces institutionnels, les circuits établis et les canons de la réussite validés par la critique appartenant au courant majoritaire en métropole ${ }^{7}$. Il ne fait aucun doute qu'il existait une concentration d'énergies collectives émergeant d'une communauté d'artistes britanniques, africaine's et asiatiques, dont les raisons et les stratégies pour faire de l'art et se confronter au canon britannique entraient fréquemment en intersection les unes avec les autres. Inévitablement, toutefois, les années 1980 virent se côtoyer au même moment des approches, des stratégies et des styles esthétiques et conceptuels différents véhiculés par un grand nombre d'artistes installée's en Grande-Bretagne.

5 De même, durant ce moment charnière que fut le colloque Shades of Black, l'historien de l'art britannique Kobena Mercer parla de « l'iconographie après l'identité » :

6 Parce que tant d'écrits [au sujet des British Black Artists] se concentrent sur l'identité biographique de l'artiste ou l'expérience de l'exclusion dans la pratique des 
institutions, les problèmes plus intéressants et les questions d'interprétation concernant l'iconographie et l'iconologie tendent à être repoussés et différés. Je voudrais montrer que les critiques et celles et ceux qui veulent à écrire sur l'art sont maintenant arrivée's à un point où il n'est plus possible de continuer de cette façon'.

7 Il ne s'agi(ssai)t pas seulement de dilemmes externes au mouvement Black Arts mais d'une problématique existant dans les discussions entre celles et ceux qui y participaient « de l'intérieur », Mercer inclus.

8 Nombre d'auteurs, souvent issus de la discipline de la sociologie héritée des cultural studies, ont contribué à construire les différentes positions des artistes du British Black Art (Stuart Hall, Hazel Carby, Paul Gilroy, Kobena Mercer) ${ }^{9}$.

9 Orlando prend clairement l'appel de Mercer comme motivation centrale pour s'engager dans sa recherche sur les British Black Artists en s'appuyant sur les méthodes structuralistes, formalistes, post-structuralistes et déconstructionnistes, avec l'idée d'« ouvrir des pistes interprétatives $»^{10}$. Il me semble qu'Orlando réussit à offrir des approches sur les British Black Artists qui diffèrent de celles des livres déjà disponibles dans ce champ.

Le livre d'Orlando, fruit de recherches précises et néanmoins accessible, apporte une contribution opportune et appréciable à ces discussions qui évoluent dorénavant avec rapidité, notamment en ce qui concerne l'intention d'établir dès le départ un dialogue critique avec des œuvres spécifiques et les tentatives de se départir d'un cadre sociologique. Elle détaille ses intentions dans l'introduction:

11 L'essai que je propose ici interroge systématiquement le rapport entre les outils plastiques propres aux productions artistiques et leur appartenance à un lieu genré, racisé et sexué et inscrit dans une classe sociales. Pour ce faire, il associe l'héritage formaliste du poststructuralisme à l'intersectionnalité. Une méthodologie qui a pour but de sortir de la lecture sociologique des artistes au profit d'une analyse des formes artistiques, tout en prenant en charge l'historicité de la construction située des savoirs académiques et des propositions plastiques ${ }^{11}$.

12 Contextualisant avec précaution sa recherche, Orlando reconnaît les complexités et les difficultés d'écrire sur une période des arts visuels compliquée d'un point de vue historique et fait montre d'un grand respect envers les œuvres et les artistes qu'elle étudie. Née en France, Orlando inscrit son livre dès le préambule entre sa propre double migration - elle hérite de deux histoires d'immigration italienne - et le terme «Black» qui connote une "posture politique commune aux migrants et enfants de migrants sur un territoire postcolonial et Européen $»^{12}$. L'auteure présente donc son livre comme une " approche intersectionnelle de l'histoire de l'art occidental » en ne soulignant pas seulement sa position bien placée comme historienne de l'art dans un domaine où la migration et la dualité existent dans de nombreuses œuvres, mais aussi en reconnaissant les caractéristiques fondamentales de l'histoire de l'art occidental, à savoir qu'elle est hiérarchique, exclusive et manque d'une perspective intersectionnelle. Je suggère ici qu'Orlando aurait peut-être pu établir en quoi sa perspective propre est une façon de comprendre l'intersectionnalité parmi une variété de nuances d'interprétations en travail. Cela aurait pu aider le lecteur ou la lectrice peu familiere de ce concept, étant entendu qu'il existe de multiples tensions et discussions, qui impliquent la "race», la sexualité, le genre, la classe, la nation, le lieu, la 
génération, et des interprétations contradictoires ou contestées de l'intersectionnalité elle-même.

Orlando a organisé sa recherche en quatre chapitres, sans suivre le fil chronologique habituel aux livres d'histoire de l'art traditionnels, mais selon des thèmes et des œuvres d'art particulières : chapitre 1, British Black Art; chapitre 2, Déconstruire et repenser les positions de l'art occidental du XXe siècle; chapitre 3, Tactiques artistiques et nouvel internationalisme; chapitre 4 , Cultures politiques des scènes artistiques britanniques.

Le chapitre 1, British Black Art, donne quelques éléments de contexte des années 1980 dans laquelle la première génération des artistes Black né·e's britanniques (ou élevé·e·s au Royaume-Uni depuis leur plus jeune âge) commença à se ménager un espace spécifique dans les annales de l'histoire de l'art britannique ${ }^{13}$. Orlando mentionne la présentation de Claudette Johnson au colloque de la Première Convention Nationale du Black Art et le rôle absolument fondamental que Johnson joua - avec d'autres artistes femmes - dans les premiers temps du «mouvement». Je me suis réjouie de voir qu'Orlando avait inséré une image de I came to dance de Johnson (1982), dont l'œuvre n'est souvent pas assez reconnue, et dont l'implication vitale dans le Blk Art Groupe et les discussions autour des British Black artists en général est souvent sous-représentée ou laissée hors champ (fig. 1) ${ }^{14}$.

Fig. 1

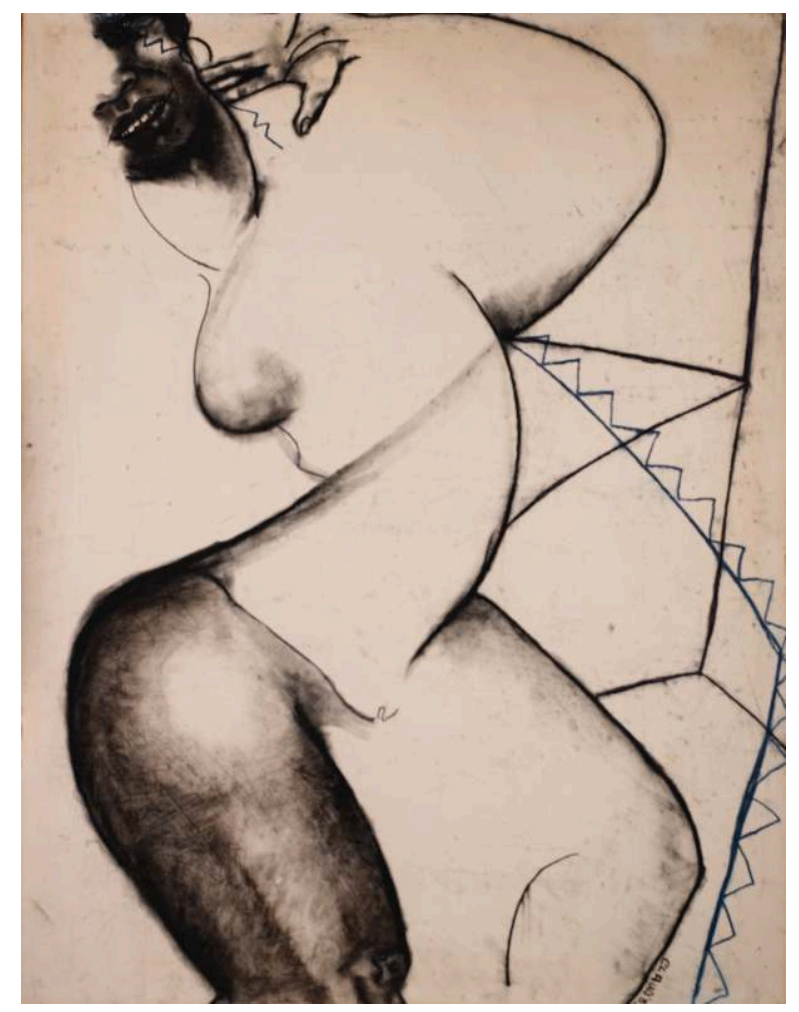

Claudette Johnson, I came to dance, pastels sur papier, 1982

(c) Claudette Johnson, Photo Denise Swanson

Le chapitre 2 - Déconstruire et repenser les positions de l'art occidental du XXe siècle se concentre sur Lubaina Himid et Eddie Chambers et "la manière don't certaines œuvres font "déconstruction" dans le sens propose par les philosophes poststructuralistes [avec les artistes] fondée sur l'analyse [...] des politiques du sujet, au 
cœur de l'histoire de la modernisation et du modernisme $»^{15}$. C'est toujours une joie de voir une attention accordée aux œuvres de Himid comme Freedom and Change (1984) et à leurs relations avec l'œuvre de Picasso (fig. 2). Orlando s'appuie sur les discussions détaillées de Freedom and Change avec le modernisme et la modernité dans l'essai de Gilane Tawadro, «Beyond the Boundary: The Work of Three Black Women Artists in Britain $»^{16}$ et commente :

[Himid] actualise le tableau de Pablo Picasso de 1922, Deux femmes courant sur la plage en déplaçant et en contextualisant le travail du peintre espagnol dans une Europe aux racines antiques qui court vers son lendemain et sa modernité [...] Elle opère ici un décentrement du sujet blanc repoussé à la marge. La côte n'y est plus celle de Dinard mais celle du commerce esclavagiste. La femme noire, et significativement deux femmes placées au centre du cadre, en appelle à une historicisation des populations noires, tout en suggérant une réflexion sur la sexualité et ses représentations au sein d'une période chargée d'une réflexion sur les identités nationales (Britishness) ${ }^{17}$.

Fig. 2

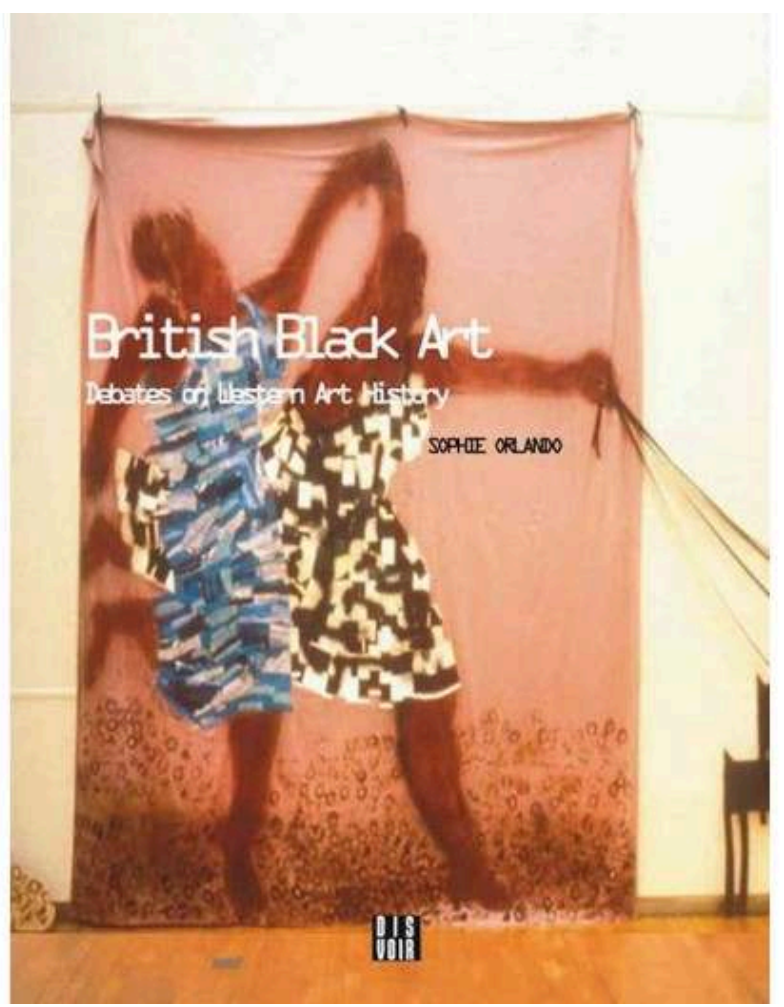

Couverture du livre de Sophie Orlando présentant Lubaina Himid, Freedom and Change, 1984

Chapitre III : Tactiques artistiques et nouvel internationalisme sur Rasheed Araeen, Sonia Boyce et Sarah Lucas ${ }^{18}$.

Ce chapitre observe les congruences entre les mutations politiques et culturelles des années 1975 et 1989 et les choix artistiques et plastiques de ces deux artistes réévalués à l'aune des notions de «cosmopolitisme», d'« internationalisme» et de "nouvel internationalisme $»^{19}$.

19 Les passages de l'auteure sur les «identités de genre ", à propos de Boyce et de Lucas, sont particulièrement remarquables ${ }^{20}$. 
Chila Kumari Burman, dont l'œuvre m'est moins familière, est abordée de manière très détaillée dans le chapitre 4 : Cultures politiques des scènes artistiques britanniques ${ }^{21}$. Orlando situe Kumari Burman comme une artiste dont la critique de la "blackness » conteste un lieu commun des études culturelles qui associent cette notion aux seulees artistes noire's. Orlando souligne que :

Les cultural studies sont donc à comprendre comme un outil de création partagée par des artistes en Grande Bretagne bien au-delà du cadre du British Black Art [...] ${ }^{22}$. Pour elle :

La traversée artistique de Chila Kumari Burman des années 1995 à 2015 puise dans les cultures politiques marxistes et féministes prévalentes dans les années 1970. Puis elle déconstruit grâce à son approche Black féministe les stéréotypes de la blackness viriliste hypersexualisée ${ }^{23}$.

Orlando remarque les influences culturelles à multiples facettes des œuvres de Kumari Burman, allant de la musique punk et du blues en passant par les théories marxiste et féministe, et la poésie afro-caribéenne ainsi que l'activisme féministe, troublant encore un peu plus les idées que l'on peut se faire sur les itinéraires culturels qui constituent l'identité d'un'e British Black artist ou la culture britannique en général. fournir de chronologie, et d'éviter qu'un nombre fini d'artistes associée's à la période puisse être résumé en un seul endroit. C'est un soulagement de le constater et une décision clé dans sa manière d'aborder le projet de British Black Art. L'histoire de l'art occidental en débat. La perspective intersectionnelle sus-mentionnée permet de plus de prendre conscience que des représentations de British Black artists, si fréquemment isolées, partagent des buts et une esthétique collectifs et spécifiques. De même Orlando souligne le nombre d'écrivains et de penseurs impliqués dans la formation d'un discours sur les British Black artists en évoquant une variété de chercheurs'ses et de champs tout au long de son livre. Elle se réfère, par exemple, non seulement à des artistes, des historien'ne's de l'art, des curateurs'rices et des critiques, mais aussi des chercheursses en histoire sociale de l'art, en études féministes, en féminisme Black, en études culturelles, en sociologie, en littérature, en politique, en postcolonialisme, en études de genre, en théorie queer et en cinéma. Cette liste sert à illustrer la connaissance d'Orlando du noyau pluraliste inhérent au British Black Art, et simultanément que cette nature pluridisciplinaire est l'une des problématiques essentielles qui méritent débat.

Orlando a retrouvé, écouté, rassemblé et présenté une sélection précise d'événements, de personnes et d'œuvres d'art; et ce faisant, elle a documenté des histoires et des pratiques de plusieurs British Black artists qui ont été et sont encore écartés des récits « officiels» et des espaces canoniques de l'histoire de l'art tels que les programmes éducatifs, la plupart des publications académiques, des monographies et des expositions rétrospectives et/ou personnelles. Il est dommage que le livre soit si court et ne donne pas à l'auteure un espace étendu pour envisager et analyser plus d'œuvres. Ceci étant dit, son choix s'avère fécond : étudier en profondeur une sélection d'artistes plutôt que de tenter d'offrir une histoire exhaustive ou un cadre délimité d'analyse critique qui définirait les "figures-clés» du mouvement. Cette stratégie exclut inévitablement un certain nombre de praticien.ne-s de la création. J'ai également apprécié la décision d'Orlando d'inclure bon nombre d'artistes qui ne sont pas 
ordinairement associés avec l'idée ou les récits du British Black Art comme Sarah Lucas, Tracey Emin et Jeremy Deller. Il aurait été rafraichissant de lire davantage d'études sur les œuvres de Himid, Burman et Chambers, tant le texte d'Orlando, du fait de son intérêt, donne envie au lecteur ou à la lectrice d'en savoir plus. Je pense qu'il est difficile, alors que les recherches commencent à peine à se diffuser largement, de répondre aux problématiques mises en avant par Hall et Mercer dans l'approche de ce moment de l'histoire de l'art. Orlando montre qu'elle a considéré avec attention ces défis tout en apportant un document débordant de noms d'artistes et de théoricien'ne's. On pourrait formuler une critique mineure - mais cruciale - à son assertion selon laquelle le Blk Art Group « fonda le mouvement » du British Black Art dans les années $1980^{24}$. S'il n'y a aucun doute que le Blk Art Groupe, et leur convention de 1982 en particulier, fut un élément dynamique fondamental du mouvement, spécialement le rôle central que les femmes artistes y jouèrent, il faut aussi ajouter d'innombrables créateurs-rices et de gens actifs à plusieurs niveaux d'implications, basés dans plusieurs lieux géographiques, avec des itinéraires variés, qui contribuèrent au développement du mouvement Black Arts. Il est significatif d'observer quelles histoires sont racontées et qui est mentionné dans ces débats récurrents, et de constater que se répètent les noms et les œuvres d'art qui commencent à être les «visages» du mouvement des British Black Arts $^{25}$. Je pense qu'il est d'autant plus important d'être attentif au genre de récits qui sont présentés dans les histoires de l'art que l'on (re)transmet. Se plaçant à côté des deux autres publications majeures dédiées à ce champ spécifique, le livre d'Orlando apporte une nouvelle perspective en (re)visitant les forces du British Black Art des années 1980 et $1990^{26}$. Shades of Black et le livre d'Eddie Chambers, Black Artists in British Art: A History Since the 1950s, publié en 2014, ont été publiés par des auteurs «internes» au mouvement Black Arts ${ }^{27}$. Orlando en revanche approche cette période de l'extérieur, comme une historienne de l'art qui a accès aux histoires via les archives et les entrevues mais sans en avoir d'expérience de première main. Shades of Black fut la première réelle tentative collective de revisiter les débats de l'époque et les problématiques autour du développement d'un discours, alors que le livre de Chambers cherche à élaborer quelques récits sur des artistes britanniques africainees et asiatiques, en résumant quelques éléments de contexte historique. Orlando prend le parti de se détacher des approches historiques et sociologiques en faveur d'« études de cas " d'œuvres et d'analyses, et réussit à produire une contribution bienvenue au corpus de la littérature sur le sujet.

Le livre d'Orlando, théoriquement puissant et soutenu par une recherche solide, évoque un grand nombre d'écrivains, penseurs, curateurs et artistes, tout en restant accessible. En tenant compte de ses limites en termes de longueur, cet essai approfondi offre au lecteur ou à la lectrice des repères pour l'inciter à développer d'autres recherches sur des événements et des activités documentés. Ce livre sera une source inestimable autant pour les étudiant·e's et les enseignante-s qui se consacrent à un grand nombre de sujets comme les beaux-arts, la photographie et le cinéma, l'histoire de l'art, les études sur les médias, la culture visuelle, l'histoire, les études culturelles, les féminismes et d'autres encore. 


\section{NOTES}

1. Sophie ORLANDO, British Black Art: Debates on Western Art History, Translated from French by Charles La Via, Paris, Dis Voir, 2016.

2. Le terme de "Black Arts Mouvement ", comme la période, n'ont pas de terminologie fixe, on les appelle aussi «British black arts movement », comme on le trouve dans le glossaire en ligne de la Tate. J'ai décidé, dans ce texte, de suivre l'interprétation d'Orlando de cette terminologie et de ce moment.

3. « [...] individuals who took on more than one role: artists who curate, write, advocate, and do archival work as well », Kobena MERCER, «Iconography after Identity », Shades of Black: Assembling Black Arts in 1980s Britain, ed. by David A. BAILEY, Ian BAUCOM, Sonia BOYCE (Durham \& London: Duke University Press, Iniva, Aavaa, 2005), p. 49-58, p. 50.

4. Sophie ORLANDO, op. cit.., p. 11.

5. Ibid.

6. Ibid., p. 17 et 7.

7. Stuart HALL, « Assembling the 1980s: The Deluge - and After ", BAILEY et al (ed.), Shades of Black, p. $1-20$, p. 2.

8. K. MERCER, Shades of Black, p. 53.

9. Sophie ORLANDO, op. cit., p. 13.

10. Ibid., p. 13

11. Ibid., p. 15.

12. Ibid., p. 7.

13. Ibid., p. 17-42.

14. J'utilise ici l'idée d'histoire replies hors de la vue en reference directe à l'hsitorienne de l'art britannique Jenny Tenant évouquand les plis de l'histoire de l'art. Jenny TENNANT JACKSON, «The Efficacity of Meta-Conceptual Performativity: Or, we just do not know what we are talking about ", Conceptual Odysseys : Passages to Cultural Analysis, ed. by Griselda Pollock (London \& New York : I. B. Tauris, 2007), p. 153-71.

15. Ibid., p. 45.

16. Gilane TAWADROS, 'Beyond the Boundary: The Work of Three Black Women Artists in Britain', Third Text, 8 (1989), p. 121-150.

17. Ibid. p. 52.

18. P. $70-100$.

19. Ibid., p. 70.

20. Ibid., p. 89.

21. Ibid., p. 101-121.

22. Ibid., p. 117.

23. Ibid., p. 121.

24. Ibid., p. 17.

25. On peut compter parmi les membres du Blk Art Group Eddie Chambers, Claudette Johnson, Keith Piper, Donald Rodney and Marlene Smith. Iels ont organisé la Première Convention Nationale du Black Art at Wolverhampton Polytechnic in 1982.

26. Les publications à venir dans ce champ comprennent: Celeste-Marie BERNIER, Stick to the Skin: Representing the Body, Memory, and History in Fifty Years of African American and Black British Art (1965-2015), University of California Press; and Anjalie DALAL-Clayton, Curating Black British Art: Exhibition Cultures Since the 1980s, I.B.Tauris (an imprint of Bloomsbury). Leon WAINWRIGHT, 
Phenomenal Difference: A Philosophy of Black British Art, Liverpool University Press, 2017, non encore publié au moment de la redaction de ce compte-rendu.

27. Eddie CHAMBERS, Black Artists in British Art: A History Since the 1950s, London: I.B. Tauris, 2014.

\section{RÉSUMÉS}

Ella S. Mills explore de nouvelles approches en Histoire de l'art, fondées sur le feminisme intersectionnel à travers le compte rendu de Sophie Orlando, British Black Art: l'histoire de l'art occidental en débat, Dis voir, Paris, 2016.

INDEX

Mots-clés : Art contemporain, Black Art, Grande-Bretagne

\section{AUTEURS}

\section{ELLA S. MILLS}

Ella S. Mills est une historienne de l'art travaillant dans le sud-ouest de l'Angleterre. Ses recherches portent sur le travail de Sutapa Biswas, Sonia Boyce, Lubaina Himid, Claudette Johnson, Ingrid Pollard and Maud Sulter. Elle développe de nouvelles approches pour les Entretiens d'Artistes fondées sur sa " méthodologie de l'écoute ", insistant sur les éléments qualitatifs d'une théorie ancrée. Elle est chercheuse en post-doctorat chargée des Entretiens d'Artistes au AHRC Black Artists and Modernist project dirigée par Sonia Boyce. Elle est aussi résidente à Kaleider, une organisation artistique à Exeter qui réunit les personnes pour imaginer, créer et produire ensemble de l'art et des expériences live.@LivingArchives @kaleider @AHRC_BAM 